\title{
The evolution of electron current sheet and formation of secondary islands in guide field reconnection
}

\author{
C. Huang ${ }^{1,2}$, Q. Lu ${ }^{1}$, Z. Yang ${ }^{1}$, M. Wu ${ }^{1}$, Q. Dong ${ }^{3}$, and S. Wang ${ }^{1}$ \\ ${ }^{1}$ CAS Key Laboratory of Basic Plasma Physics, Department of Geophysics and Planetary Sciences, University of Science and \\ Technology of China, Hefei 230026, China \\ ${ }^{2}$ State Key Laboratory of Space Weather, Chinese Academy of Sciences, Beijing 100190, China \\ ${ }^{3}$ Beijing National Laboratory of Condensed Matter Physics, Institute of Physics, Chinese Academy of Sciences, \\ Beijing 100080, China
}

Received: 12 July 2011 - Revised: 10 October 2011 - Accepted: 11 October 2011 - Published: 20 October 2011

\begin{abstract}
Two-dimensional (2-D) particle-in-cell (PIC) simulations are performed to investigate the evolution of the electron current sheet (ECS) in guide field reconnection. The ECS is formed by electrons accelerated by the inductive electric field in the vicinity of the $\mathrm{X}$ line, which is then extended along the $x$ direction due to the imbalance between the electric field force and Ampere force. The tearing instability is unstable when the ECS becomes sufficiently long and thin, and several seed islands are formed in the ECS. These tiny islands may coalesce and form a larger secondary island in the center of the diffusion region.
\end{abstract}

\section{Introduction}

Magnetic reconnection is a fundamental physical process in plasma, which provides an effective mechanism for fast energy conversion from magnetic energy to plasma kinetic and thermal energies (Vasyliunas, 1975; Biskamp, 2000; Priest, 2000). It is believed that magnetic reconnection plays an important role in many explosive phenomena, which occurs in space and laboratory plasma, such as solar flares in the corona (Giovanelli, 1946), the heating of solar corona (Ulmschneider et al., 1991), substorms in the Earth's magnetosphere (Nishida, 1978), and disruptions in fusion experiments (Wesson, 1997).

Hall effect is considered to be a critical ingredient in collisionless magnetic reconnection. At the scale length between the ion inertial length $c / \omega_{\mathrm{pi}}$ (where $c$ and $\omega_{\mathrm{pi}}$ are the

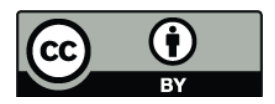

Correspondence to: $\mathrm{Q}$. $\mathrm{Lu}$

(qmlu@ustc.edu.cn) light speed and ion plasma frequency) and electron inertial length $c / \omega_{\text {pe }}$ (where $\omega_{\text {pe }}$ is the electron plasma frequency), only electrons are frozen in magnetic field lines, and ions can move across magnetic field lines (Sonnerup, 1979; Terasawa, 1983; Birn et al., 2001; Shay et al., 2001; Lu et al., 2010; Wang et al., 2010a). This region is called as ion diffusion region, and the ion-electron decoupling causes Hall effect. At the scale length below $c / \omega_{\text {pe }}$, the electron frozenin constraint is also broken, and the motions of electrons are demagnetized. Both ions and electrons can move across the magnetic field lines (Birn et al., 2001; Ma et al., 2001; Pritchett, 2001). The region is called as electron diffusion region (EDR). Therefore, magnetic reconnection is considered to be of multiscale features - a small EDR is embedded in a larger ion diffusion region.

In the EDR, electrons can be accelerated effectively. At last, the current is carried mainly by the electrons, and an electron current sheet (ECS) is formed (Fujimoto, 2006). Recently, by performing two-dimensional (2-D) particle-in-cell (PIC) simulations with open boundary conditions, Daughton et al. (2006) found that in anti-parallel reconnection the length of the ECS can be extended to tens of ion inertial lengths and the reconnection rate is reduced during the extension. The fast reconnection is realized by the following impulsive excitation of the tearing instability that generates magnetic islands in the ECS. The extension of the ECS and the evolution of the reconnection rate have also been studied in a large scale system with periodic boundary conditions (Fujimoto, 2006; Daughton, 2007). The EDR in antiparallel reconnection is composed of a diffusion region and elongated electron jets, which reachs a length tens of ion inertial lengths (Karimabadi et al., 2007; Shay et al., 2007; Hesse et al., 2008, Zenitani et al. 2011). The super-Alfvenic

Published by Copernicus Publications on behalf of the European Geosciences Union and the American Geophysical Union. 

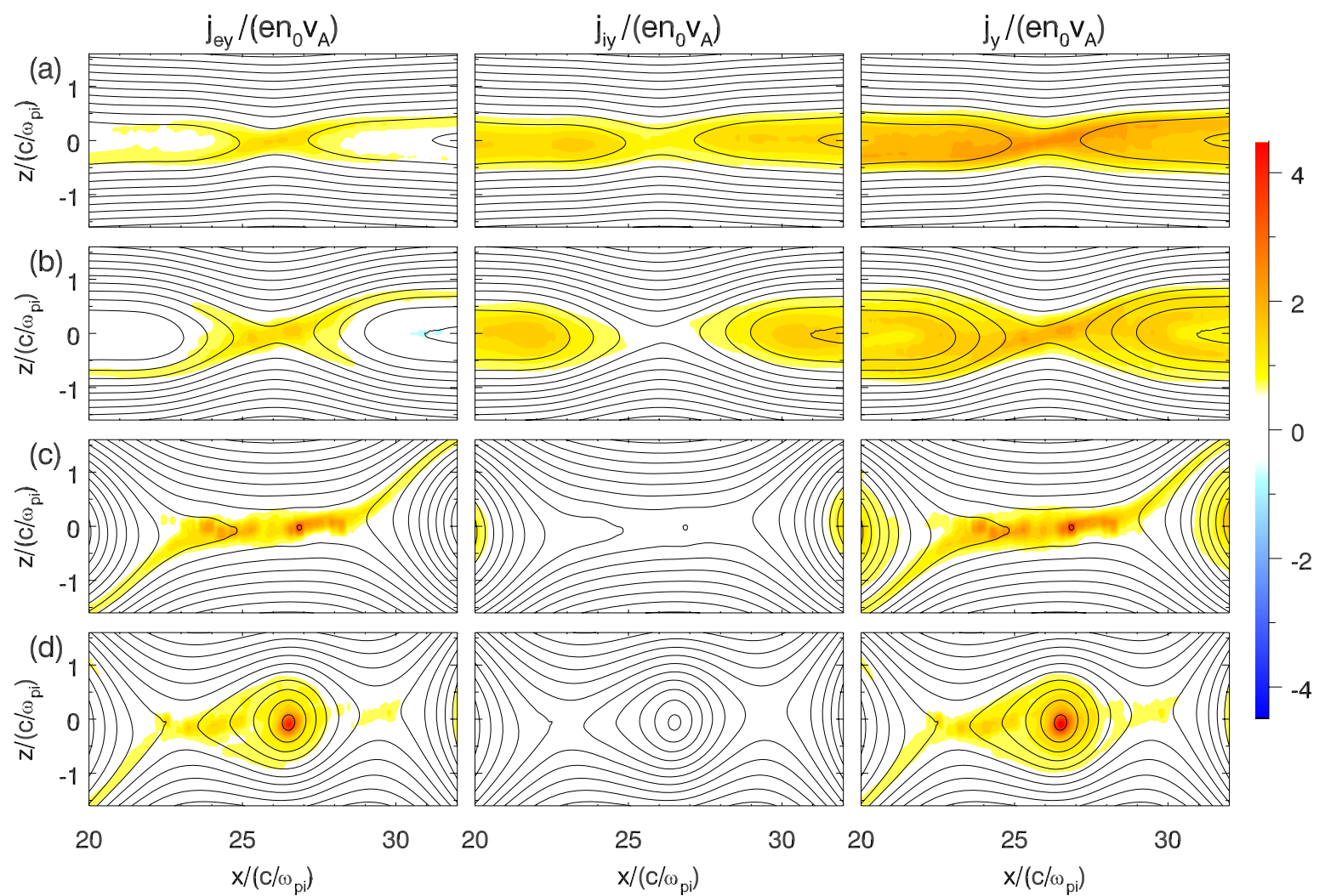

Fig. 1. The time evolution of the current sheet around $x=26.6 c / \omega_{\mathrm{pi}}$ at $\Omega_{\mathrm{i}} t=$ (a) 18 , (b) 22 , (c) 29 and (d) 36 , respectively. The left, middle and right panels describe the electron current $j_{\mathrm{e} y}$, ion current $j_{\mathrm{i} y}$ and total current $j_{y}$. The magnetic field lines (solid lines) are also plotted for reference

electron flow jets in the EDR can be explained by a combination of electric field drifts and of diamagnetic effects through the combination of the gradients of particle pressure and of the magnetic field (Hess et al., 2008).

The extension of the ECS, as well as the formation of secondary islands can also been found in guide field reconnection (Drake et al., 2005, 2006; Daughton et al., 2011). Drake et al. (2006) found that in guide field reconnection, the current layer develops a pronounced tilt as electrons accelerated parallel to the magnetic field stream preferentially outward along two of the four separatrices connected to the $x$-line. With the extension of ECS, it is unstable to the tearing instability, which then forms secondary islands. Secondary islands not only can affect reconnection rate (Daughton et al., 2006) and energize electrons effectively (Wang et al., 2010b; Oka et al., 2010), but also they may be related to the bursty nature of reconnection (Drake et al., 2006). Recently, Daughton et al. (2011) studied the three-dimensional (3-D) evolution of guide field reconnection. The evolution is dominated by the formation and interaction of helical magnetic structures known as flux ropes, which leads to a turbulent evolution where electron dynamics plays a critical role.
In this paper, by performing 2-D PIC simulations, we will further investigate more quantitatively how a current sheet is developed during guide field reconnection. It is found that the current sheet extends due to the imbalance between the electric field force and Ampere force, and the current is dominated by the electrons before it is unstable to the tearing instability and forms secondary islands. The paper is organized as follows. The simulation model is presented in Sect. 2, and the simulation results are described in Sect. 3. Section 4 gives discussion and conclusions.

\section{Simulation model}

A 2-D PIC code is used in this paper to investigate the evolution of the ECS and the formation of the secondary magnetic island in guide field reconnection. In the simulation, the electromagnetic fields are defined on the grids and updated by solving the Maxwell equations with a full explicit algorithm (Birdsall and Langdon, 1985; Pritchett, 1985). The initial configuration is a one-dimensional Harris current sheet in the $(x, z)$ plane, and the initial magnetic field is given by (Harris, 1962) 

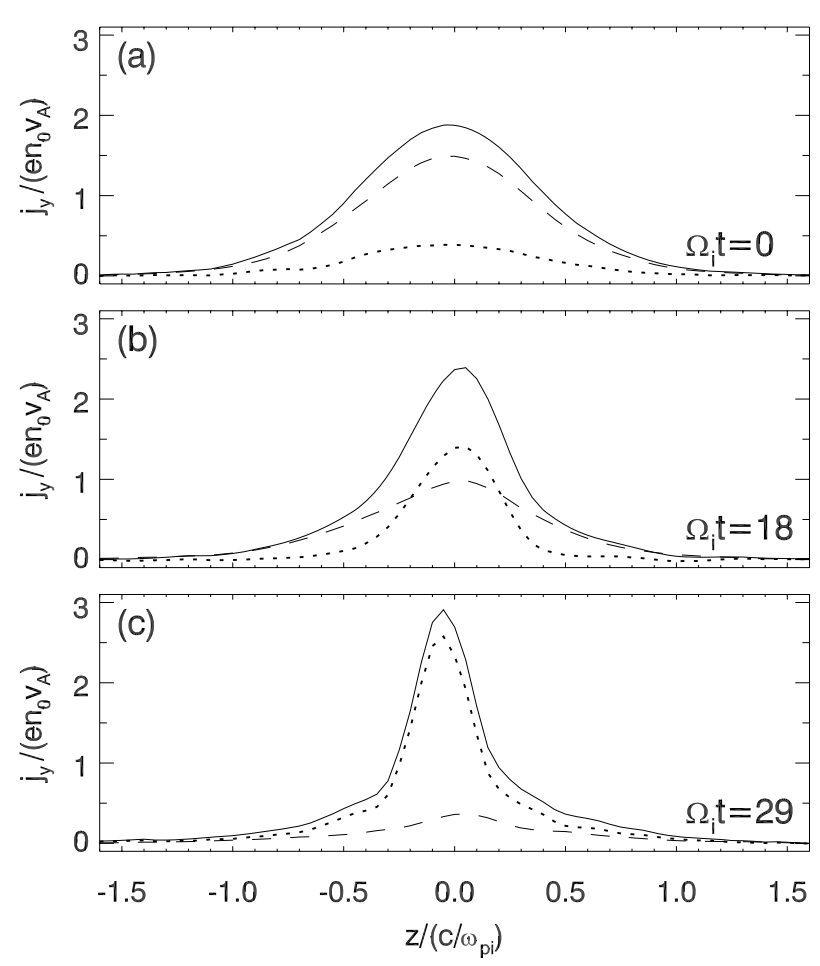

Fig. 2. The profiles of the current sheet along $x=26.6 c / \omega_{\text {pi }}$ at (a) $\Omega_{\mathrm{i}} t=0$, (b) $\Omega_{\mathrm{i}} t=18$ and (c) $\Omega_{\mathrm{i}} t=29$. The dashed, dotted and solid lines present the electron current $j_{\mathrm{e} y}$, the ion current $j_{\mathrm{i} y}$ and the total current $j_{y}$, respectively.

$\boldsymbol{B}_{0}(z)=B_{0} \tanh (z / \delta) \boldsymbol{e}_{x}+B_{y 0} \boldsymbol{e}_{y}$

where $\delta$ is the half-width of the current sheet. $B_{0}$ is the asymptotical magnetic strength. $B_{y 0}$ is the amplitude of the guide field. The corresponding number density is

$n(z)=n_{\mathrm{b}}+n_{0} \sec h^{2}(z / \delta)$,

where $n_{\mathrm{b}}$ represents the density of the background plasma and $n_{0}$ is the peak Harris density. The distribution functions for the ions and electrons are Maxwellian, and their drift speeds in the $y$ direction satisfy $V_{\mathrm{i} 0} / V_{\mathrm{e} 0}=-T_{\mathrm{i} 0} / T_{\mathrm{e} 0}$, where $V_{\mathrm{i} 0}\left(V_{\mathrm{e} 0}\right)$ and $T_{\mathrm{i} 0}\left(T_{\mathrm{e} 0}\right)$ are the initial drift speed and temperature for ions (electrons), respectively. In our simulations, the temperature ratio is $T_{\mathrm{i} 0} / T_{\mathrm{e} 0}=4$, and $n_{0}=5 n_{\mathrm{b}}$. The half-width of the current sheet is $\delta=0.5 c / \omega_{\text {pi }}$, where $c / \omega_{\text {pi }}$ is the ion inertial length defined by $n_{0}$. The mass ratio is set to $m_{\mathrm{i}} / m_{\mathrm{e}}=100$ (where $m_{\mathrm{e}}$ is the rest mass of the electron). The light speed is $c=15 v_{\mathrm{A}}$, where $v_{\mathrm{A}}$ is the Alfven speed defined by $B_{0}$ and $n_{0}$. An initial guide field $B_{y 0}=-B_{0}$ is used in the simulations.

The computation is carried out in a rectangular domain in the $(x, z)$ plane with dimension $L_{x} \times L_{z}=$ $\left(51.2 c / \omega_{\text {pi }}\right) \times\left(12.8 c / \omega_{\text {pi }}\right)$. An $N_{x} \times N_{z}=1024 \times 256$ grid system is employed in the simulations, so the spatial resolution is $\Delta x=\Delta z=0.05 c / \omega_{\mathrm{pi}}=0.5 c / \omega_{\mathrm{pe}}$. The time step is

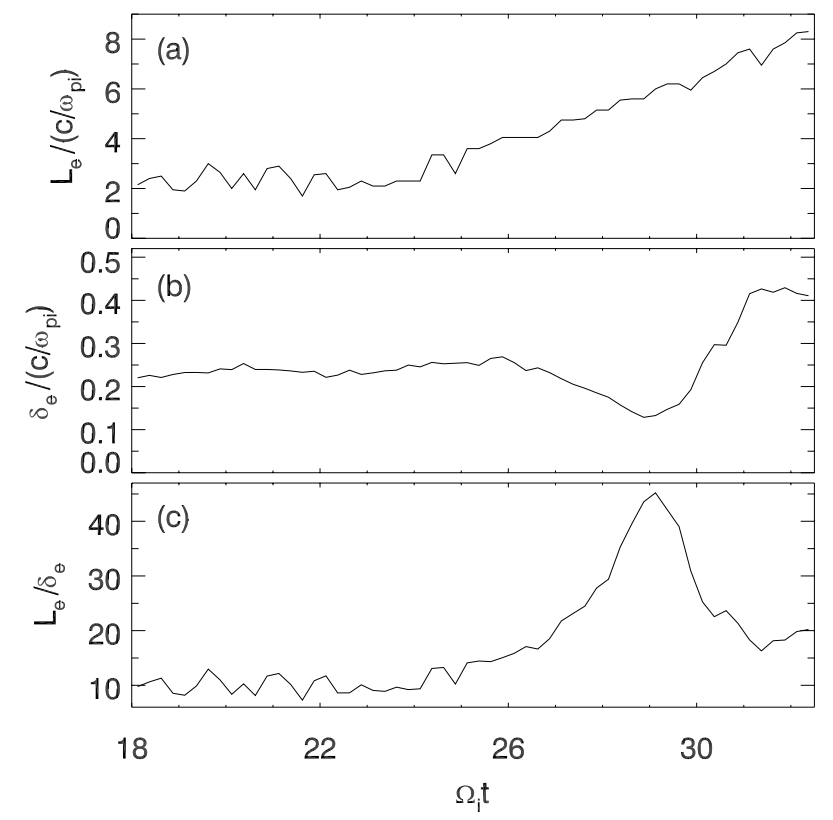

Fig. 3. The time evolutions of the characteristics of the ECS, (a) the length $L_{\mathrm{e}}$, (b) the half-width $\delta_{\mathrm{e}}$, and (c) the ratio of the length to the half-width $L_{\mathrm{e}} / \delta_{\mathrm{e}}$.

$\Omega_{\mathrm{i}} \Delta t=0.001$, where $\Omega_{\mathrm{i}}$ is the ion gyrofrequency. We employ more than $1.0 \times 10^{7}$ particles per species in the simulations. The periodic boundary conditions are used along the $x$ direction, while the ideal conducting boundary conditions for electromagnetic fields are employed in the $z$ direction. An initial flux perturbation is introduced in the simulations.

\section{Simulation results}

Figure 1 shows the time evolution of the current sheet around $x=26.6 c / \omega_{\text {pi }}$ at $\Omega_{\mathrm{i}} t=$ (a) 18 , (b) 22, (c) 29 and (d) 36, respectively. In the figure, the left, middle and right panels describe the electron current $j_{\mathrm{e} y}$, ion current $j_{\mathrm{i} y}$ and total current $j_{y}$, and the magnetic field lines are also plotted for reference. Initially, the current is carried mainly by ions, therefore, the ion current $j_{\mathrm{i} y}$ is much larger than the electron current $j_{\mathrm{e} y}$. With the proceeding of the reconnection, both ions and electrons are ejected from the vicinity of the $\mathrm{X}$ line. However, the electrons can be accelerated by the inductive electric field in the $y$ direction around the $X$ line (Huang et al., 2010). From about $\Omega_{\mathrm{i}} t=20$, the current is dominated by the electron current, and the current sheet becomes an ECS. Then, the ECS is extended along the $\mathrm{x}$ direction. When the ECS becomes sufficiently long, the sheet is unstable to the tearing instability. By the end of the simulation, a secondary island remains near the center of the diffusion region and is bounded by two X-lines.

The formation and extension of the ECS around $x=$ $26.6 c / \omega_{\text {pi }}$ can be depicted more clearly in Figs. 2 and 3. 


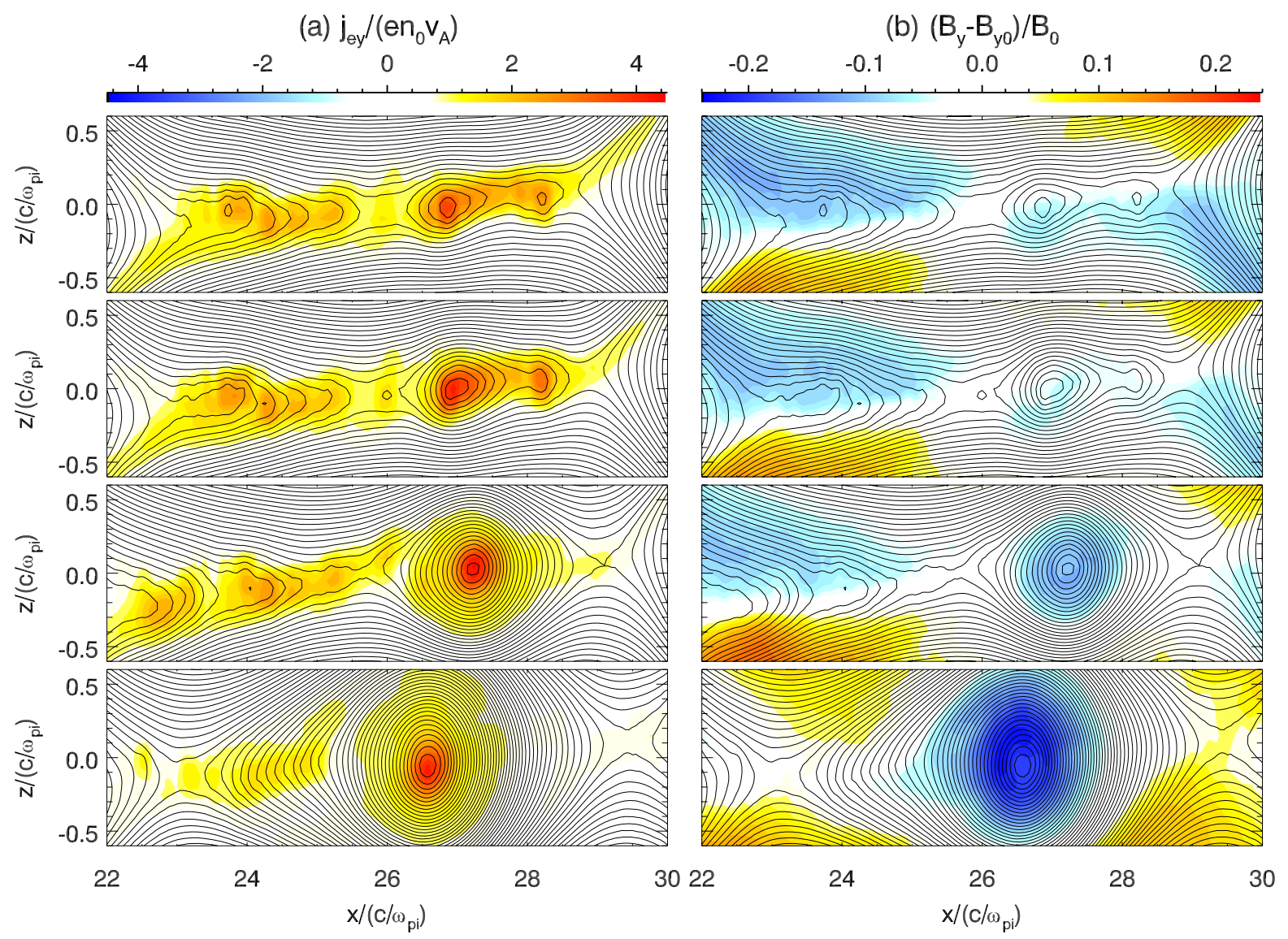

Fig. 4. The contours of (a) the electron currents along the $y$ direction $j_{\mathrm{e} y}$, and (b) the out-of-plane magnetic field $\left(B_{y}-B_{y 0}\right) / B_{0}$ at $\Omega_{\mathrm{i}} t=29$, 29.5, 30.5 and 33.5 from the top to the bottom panels. The magnetic field lines (solid lines) are also represented in the figure.

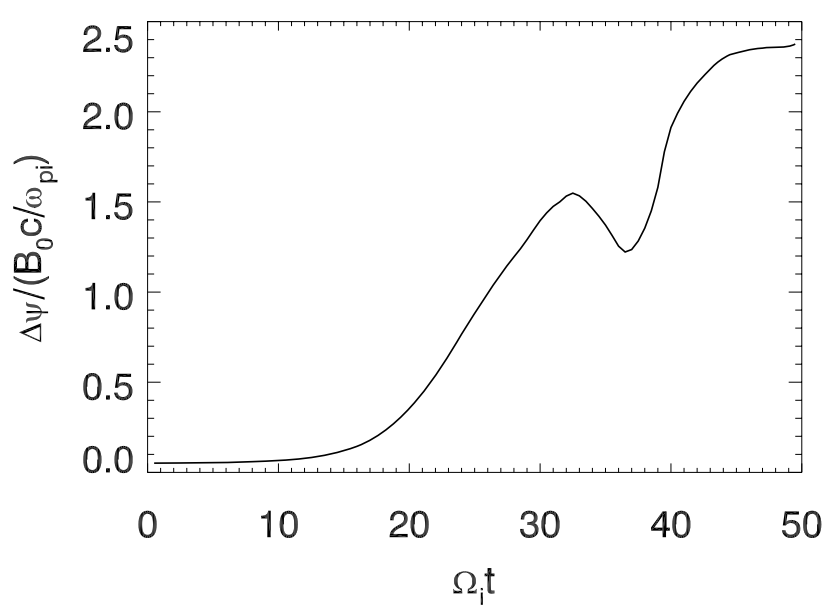

Fig. 5. Time evolution of the flux difference $\Delta \psi$ in the reconnection, where the magnetic flux $\Delta \psi$ is defined as the flux difference between the position $\left(26.6 c / \omega_{\text {pi }}, 0\right)$ and the first $\mathrm{O}$ line formed during the reconnection.
Figure 2 shows the profiles of the electron current $j_{\mathrm{e} y}$ (dotted lines), ion current $j_{\mathrm{iy}}$ (dashed lines) and total current $j_{y}$ (solid lines) along $x=26.6 c / \omega_{\mathrm{pi}}$ at (a) $\Omega_{\mathrm{i}} t=0$, (b) $\Omega_{\mathrm{i}} t=18$ and (c) $\Omega_{\mathrm{i}} t=29$. Figure 3 depicts the evolution of the characteristics of the ECS, (a) the length $L_{\mathrm{e}}$, (b) the half-width $\delta_{\mathrm{e}}$, and (c) the ratio of the length to the half-width $L_{\mathrm{e}} / \delta_{\mathrm{e}}$. The length $L_{\mathrm{e}}$ and the half-width $\delta_{\mathrm{e}}$ of the ECS are properly determined from the simulation results at the given times. The length $L_{\mathrm{e}}$ of the ECS is defined as the distance between the two peaks of the electron outflow speed along $z=0$. The profile of the ECS is assumed to satisfy Eq. (2), and then the halfwidth $\delta_{\mathrm{e}}$ is calculated by fitting the current sheet with a least square method after averaging current along the $x$ direction within the length of one ion inertial length around the $\mathrm{X}$ line. Initially, the current is carried mainly by ions. With the proceeding of the reconnection, the current become dominated by electrons, and the ECS is formed. At about $\Omega_{\mathrm{i}} t=25$, the length of the ECS increases rapidly, while the width of the ECS decrease. Therefore, the ratio $L_{\mathrm{e}} / \delta_{\mathrm{e}}$ also increases rapidly. When the ratio $L_{\mathrm{e}} / \delta_{\mathrm{e}}$ is sufficiently large, the ECS is unstable to the tearing instability. This can be demonstrated more clearly in Fig. 4, which shows the evolution of 

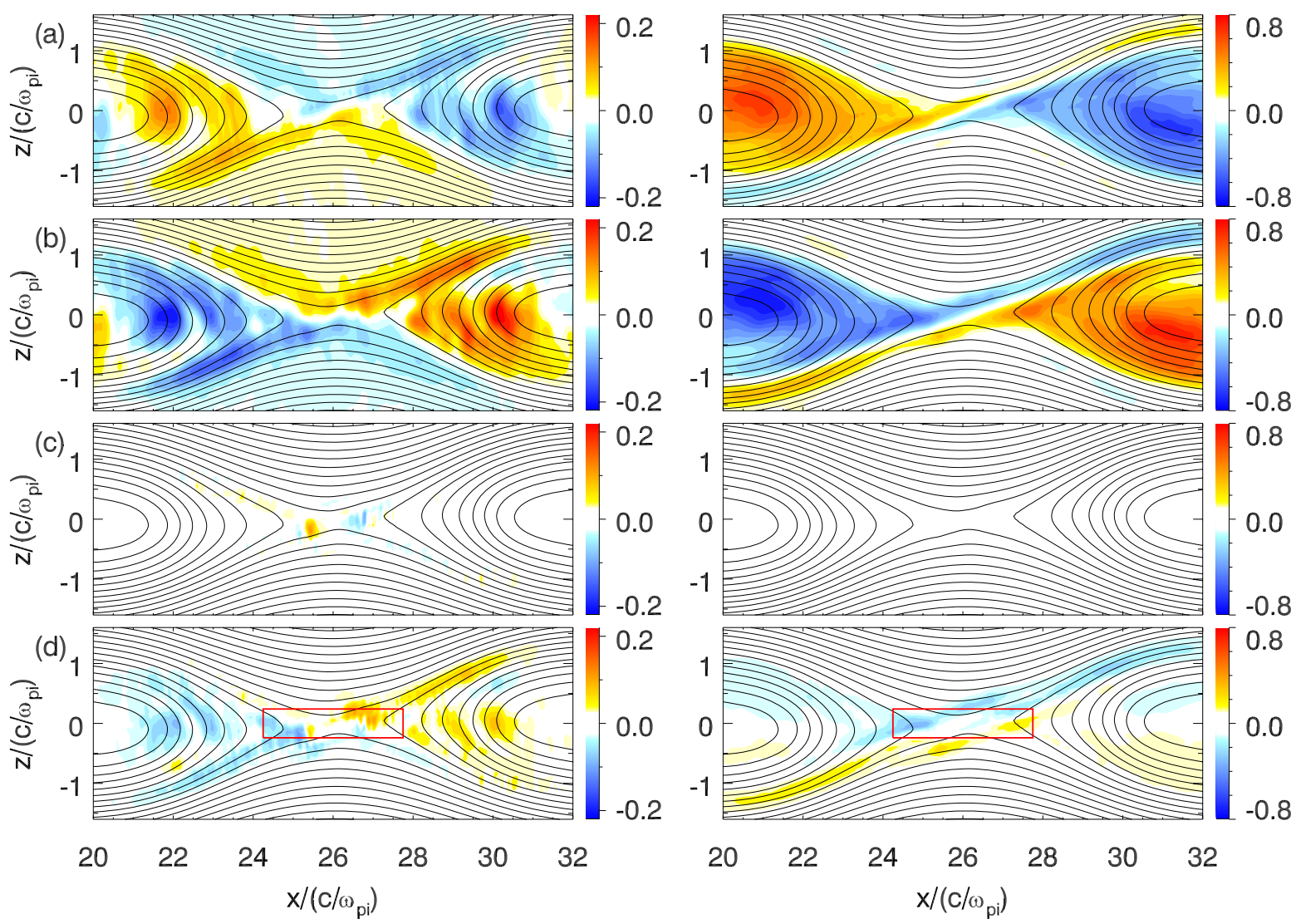

Fig. 6. (a) The electric field force $-\mathrm{en}_{\mathrm{e}} \boldsymbol{E}$, (b) the Ampere force $\boldsymbol{j}_{\mathrm{e}} \times \boldsymbol{B},(\mathbf{c})$ the pressure gradient $-\nabla \cdot \mathbf{P}_{\mathrm{e}}$, and (d) the sum of the above three forces at $\Omega_{\mathrm{i}} t=25$. The left (right) panels show the $x(z)$ component of the forces.

the seed islands formed in the ECS during the tearing instability. Figure $4 \mathrm{a}$ and $\mathrm{b}$ shows the electron currents in the $y$ direction $j_{\mathrm{e} y}$ and out of plane magnetic field $\left(B_{y}-B_{0}\right) / B_{0}$ at $\Omega_{\mathrm{i}} t=29,30.5,30$ and 33.5 from the top to the bottom panel. At $\Omega_{\mathrm{i}} t=29$, there exist three seed islands. The two seed islands in the right coalesce into one island at about $\Omega_{\mathrm{i}} t=30.5$, while the seed island in the left is expelled into the left outflow region. At $\Omega_{\mathrm{i}} t=33.5$, only a secondary island remains, where the out-of-plane magnetic field is obviously enhanced. At last, the secondary magnetic island is also expelled into the left out-flow region (not shown). According to the linear theory (Wang et al., 1990), the tearing instability is unstable in a current sheet when $2 \pi \delta_{\mathrm{e}}<\lambda$ (where $\lambda$ is the wavelength of the tearing instability). In our simulations, we can calculate that during the linear growth stage of the tearing instability the average length of the island is about $2 c / \omega_{\mathrm{pi}}$, and the half-width of the ECS $\delta_{\mathrm{e}}$ is about $0.15 c / \omega_{\mathrm{pi}}$. The condition of the tearing instability is satisfied, as described by Daughton et al. (2006).

The influence of the evolution of the ECS on the reconnection rate can be demonstrated in Fig. 5, which shows the time evolution of the flux difference $\Delta \psi$. Here $\Delta \psi$ is defined as the flux difference between $\left(x=26.6 c / \omega_{\mathrm{pi}}, z=0\right)$ and the first $\mathrm{O}$ line formed during the reconnection, and its slope can be served as an indicator of the magnetic reconnection rate. Similar to the results obtained by Daughton et al. (2006), during the extension of the ECS, the reconnection rate reduces significantly, which is then raised with the formation of the secondary island.

The extension of the ECS can be demonstrated by analyzing the exerted forces on the ECS. Figure 6 shows (a) the electric field force $-\mathrm{en}_{\mathrm{e}} \boldsymbol{E}$, (b) the Ampere force $\boldsymbol{j}_{\mathrm{e}} \times \boldsymbol{B}$, (c) the pressure gradient $-\nabla \cdot \mathbf{P}_{\mathrm{e}}$, (d) the sum of the above three forces at $\Omega_{\mathrm{i}} t=25$, the left and right panels plot the $x$ and $z$ components, respectively. The effect of the pressure gradient can be neglected. The electric field force tries to squeeze the ECS in the $x$ direction, and stretch the ECS in the $z$ direction. The Ampere force is stretching the ECS in the $x$ direction and squeezing the ECS in the $z$ direction. The amplitude of the Ampere force is larger than that of the electric field force. Therefore, the resulted ECS is extended along the $x$ direction and squeezed in the $z$ direction, which results in a long and thin ECS. 


\section{Discussion and conclusions}

The evolution of the electron current sheet is considered to play a critical role in determining the reconnection rate in collisionless magnetic reconnection. In anti-parallel reconnection, the ECS is found to be extended to tens of ion inertial lengths, and the reconnection rate is reduced significantly during the extension. The fast reconnection is realized by the following impulsive excitation of the tearing instability that generate secondary islands in the ECS (Daughton et al., 2006). In this paper, with 2-D PIC simulations, we investigate the evolution of the electron current sheet in guide field reconnection. The ECS is found to be formed due to the energetic electrons accelerated by the inductive electric field in the vicinity of the $\mathrm{X}$ line. Then the ECS is extended along the $x$ direction exerted by the Ampere force. At last, the ECS is unstable to the tearing instability, and a secondary island is formed in the center of the ion diffusion region at last. The out-of-plane magnetic field is enhanced in the secondary island, which is consistent with the Cluster observations (Wang et al., 2010b).

Acknowledgements. This research was supported by the National Science Foundation of China, Grant Nos. 40931053, 40725013, 40974081, Ocean Public Welfare Scientific Research Project, State Oceanic Administration People's Republic of China (No. 201005017), the Specialized Research Fund for State Key Laboratories, and the Fundamental Research Funds for the Central Universities (WK2080000010).

Edited by: G. Lapenta

Reviewed by: Z. Voros and another anonymous referee

\section{References}

Birdsall, C. K. and Langdon, A. B.: Plasma Physics via Computer Simulation, Mcgraw-Hill Book Company, 1985.

Birn, J., Drake, J. F., Shay, M. A., Rogers, B. N., Denton, R. E., Hesse, M., Kuznetsova, M., Ma, Z. W., Bhattacharjee, A., Otto, A., and Pritchett, P. L.: Geospace Environmental Modeling (GEM) Magnetic Reconnection Challenge, J. Geophys. Res., 106, 3715, doi:10.1029/1999JA900449, 2001.

Biskamp, D.: Magnetic Reconnection in Plasmas, Cambridge Univ. Press, Cambridge, UK, 2000.

Daughton, W.: Surprising new results on the structure of the diffusion region, Eos Trans. AGU, 88, Jt. Assem. Suppl., Abstract SM53C-04, 2007.

Daughton, W., Scudder, J., and Karimabadi, H.: Fully kinetic simulations of undriven magnetic reconnection with open boundry conditions, Phys. Plasmas, 13, 072101, doi:10.1063/1.2218817, 2006.

Daughton, W., Roytershteyn, V., Karimabadi, H., Yin, L., Albright, B. J., Bergen, B., and Bowers, K. J.: Role of electron physics in the development of turbulent magnetic reconnection in collisionless plasmas, Nature Phys., 7, 539-542, doi:10.1038/nphys1965, 2011.
Drake, J. F., Shay, M. A., and Thongthai, W.: Production of Energetic Electrons during Magnetic Reconnection, Phys. Rev. Lett., 94, 095001, doi:10.1103/PhysRevLett.94.095001, 2005.

Drake, J. F., Swisdak, M., Schoeffler, K. M., Rogers, B. N., and Kobayashi, S.: Formation of secondary islands during magnetic reconnection, Geophys. Res. Lett., 33, L13105, doi:10.1029/2006GL025957, 2006.

Fujimoto, K.: Time evolution of the electron diffusion region and the reconnection rate in fully kinetic and large system, Phys. Plasmas, 13, 072904, doi:10.1063/1.2220534, 2006.

Giovanelli, R. G.: A theory of chromospheric flares, Nature, 158, 81-82, doi:10.1038/158081a0, 1946.

Harris, E. G.: On a plasma sheath separating regions of oppositely directed magnetic field, Nuovo Cimento Soc. Ital. Fis., 23, 115121, doi:10.1007/BF02733547, 1962.

Hesse, M., Zenitani, S., and Klimas, A.: The structure of electron outflow jet in collisionless magnetic reconnection, Phys. Plasmas, 15, 112102, doi:10.1063/1.3006341, 2008.

Huang, C., Lu, Q. M., and Wang, S.: The mechanisms of electron acceleration in antiparallel and guide field magnetic reconnection, Phys. Plasmas, 17, 072306, doi:10.1063/1.3457930, 2010.

Karimabadi, H., Daughton, W., and Scudder, J.: Multi-scale structure of the electron diffusion region, Geophys. Res. Lett., 34, L13104, doi:10.1029/2007GL030306, 2007.

Lu, Q. M., Huang, C., Xie, J. L., Wang, R. S., Wu, M. Y., Vaivads, A., and Wang, S.: Features of separatrix regions in magnetic reconnection: Comparison of 2-D particle-in-cell simulations and Cluster observations, J. Geophys. Res., 115, A11208, doi:10.1029/2010JA015713, 2010.

Ma, Z. W. and Bhattacharjee, A.: Hall magnetohydrodynamic reconnection: The Geospace Environment Modeling challenge, J. Geophys. Res., 106, 3773, doi:10.1029/1999JA001004, 2001.

Nishida, A.: Geomagnetic Diagnostics of the Magnetosphere, Springer, New York, 1978.

Oka, M., Fujimoto, M., Shinohara, I., and Phan T. D.: "Island surfing" mechanism of electron acceleration during magnetic reconnection, J. Geophys. Res., 115, A08223, doi:10.1029/2010JA015392, 2010.

Priest, E. and Forbes, T.: Magnetic Reconnection: MHD Theory and Applications, Cambridge Univ. Press, Cambridge, UK, 2000.

Pritchett, P. L.: Electromagnetic particle simulation codes, Space Sci. Rev., 42, 17-27, doi:10.1007/BF00218220, 1985.

Pritchett, P. L.: Geospace Environment Modeling magnetic reconnection challenge: Simulations with a full particle electromagnetic code, J. Geophys. Res., 106, 3783, doi:10.1029/1999JA001006, 2001.

Sonnerup, B. U. Ö.: Magnetic field reconnection, in Solar System Plasma Physics, edited by: Lanzèerotti, L. J., Kennel, C. F., and Parker, E. N., North-Holland, New York, vol. 3, p. 46, 1979.

Shay, M. A., Drake, J. F., Rogers, B. N., and Denton, R. E.: Alfvénic collisionless magnetic reconnection and the Hall term, J. Geophys. Res., 106, 3759, doi:10.1029/1999JA001007, 2001.

Shay, M. A., Drake, J. F., and Swisdak, M.: Two-scale structure of the electron dissipation region during collisionless magnetic reconnection, Phys. Rev. Lett., 99, 155002, doi:10.1103/PhysRevLett.99.155002, 2007.

Terasawa, T.: Hall current effect on tearing mode instability, Geophys. Res. Lett., 10, 475-478, 1983. 
Ulmschneider, P., Priest, E. R., and Rosner, R.: Mechanisms of Chromospheric and Coronal Heating, edited by: Rosner, R., Springer, Berlin, 1991.

Vasyliunas, V. M.: Theoretical models of magnetic field line merging, Rev. Geophys. Space Phys., 13, 303-336, 1975.

Wang, R. S., Lu, Q. M., Huang, C., and Wang, S.: Multispacecraft observation of electron pitch angle distributions in magnetotail reconnection, J. Geophys. Res., 115, A01209, doi:10.1029/2009JA014553, 2010a.

Wang, R. S., Lu, Q. M., Du, A. M., and Wang, S.: In situ observations of a secondary magnetic island in ion diffusion region and associated energetic electrons, Phys. Rev. Lett., 104, 175003, doi:10.1103/PhysRevLett.104.175003, 2010b.
Wang, X. and Bhattacharjee, A.: Collisionless tearing instability in magnetic plasma, J. Geophys. Res., 95, 15047-15057, 1990.

Wesson, J.: Tokomaks, Oxford Univ. Press, New York, 1997.

Zenitani, S., Hesse, M., Klimas, A., and Kuznetsova, M.: New Measure of the Dissipation Region in Collisionless Magnetic Reconnection, Phys. Rev. Lett., 106, 195003, doi:10.1103/PhysRevLett.106.195003, 2011. 